\title{
Optimizing Waveforms for Positioning in 5G
}

\author{
Armin Dammann, Thomas Jost, Ronald Raulefs, Michael Walter, Siwei Zhang \\ Institute of Communications and Navigation, German Aerospace Center (DLR) \\ Oberpfaffenhofen, 82234 Wessling, Germany \\ Email: \{Armin.Dammann, Thomas.Jost, Ronald.Raulefs, M.Walter, Siwei.Zhang\}@DLR.de
}

\begin{abstract}
Today's mobile radio systems deploy reference signals which can be used or which are even dedicated for signal propagation delay-based mobile terminal positioning. Usually, the signal power of such reference signals is uniformly distributed over the available spectrum. It is known from estimation theory that such a uniform power distribution of reference signals is not optimal for signal propagation delay estimation.

In this paper we consider mobile terminal positioning based on signal propagation delay estimation in the uplink case. For positioning, we introduce a parametric waveform. This waveform provides a scalar parameter for controlling the distribution of the available signal power over the spectrum. Using this waveform parameter we aim to minimize the positioning error. For optimization, we require a functional dependency between the waveform parameter and the positioning error we can expect. For the derivation of this function we combine the approaches of the Cramér-Rao and Ziv-Zakai bounds for position and propagation delay estimation. As an exemplary environment we consider a mobile terminal located in an area surrounded by 3 base stations. For this environment we show that the optimized waveform spends a significant part of the available power at the spectrum edges, leading to a performance gain of $37.3 \%$ at the center of the area between the base stations.
\end{abstract}

\section{INTRODUCTION}

The current deployed standard of cellular mobile radio systems is the 4th generation called Long-Term Evolution (LTE). Up to its Release 9, LTE defined a single time-based procedure to estimate the range between a mobile and a base station. The range based procedure is called observed time difference of arrival (OTDOA) and calculates the position through the observed range based estimates of the mobile terminal in the downlink. In Release 11 uplink TDOA (UTDOA) was standardized. In this paper we present an analysis that is based on uplink signals and is aligned with the current discussion about the future waveform of the next generation cellular mobile radio $(5 \mathrm{G})$ for communication needs.

Currently, the question how a communication system of the $5^{\text {th }}$ generation will look like is intensely discussed. Insights into research and development towards $5 \mathrm{G}$ can be found for example in [1]-[3]. The trend here is clearly that $5 \mathrm{G}$ is not an incremental evolution of LTE. By using new technology and paradigms as well as seamless integration of radio access technologies $5 \mathrm{G}$ aims to provide 10-100x higher user data rate, 1000x higher mobile data volume per area, 10-100x higher number of connected devices, 10x longer battery lifetime and $5 \mathrm{x}$ reduced end-to-end latency [3]. Besides requirements related to communications, mentioned above, the authors of [4] have addressed that in $5 \mathrm{G}$ network based positioning should be supported with accuracy of $10 \mathrm{~m}$ down to less than $1 \mathrm{~m}$ in $80 \%$ of occasions and less than $1 \mathrm{~m}$ indoors. Several technologies and properties envisaged for $5 \mathrm{G}$ in order to meet the chal-

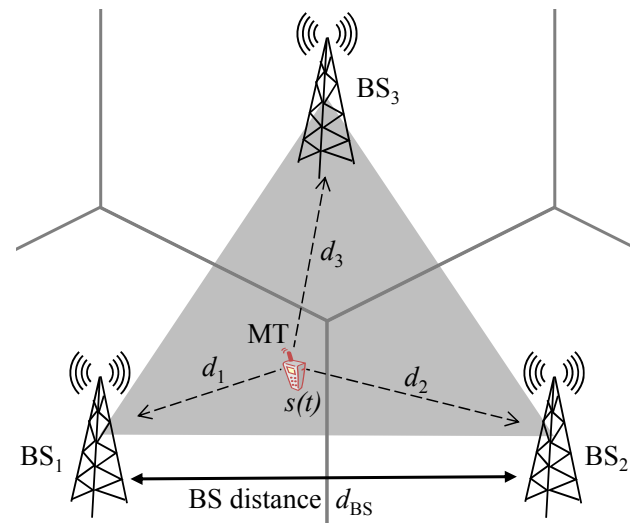

Figure 1. Mobile terminal positioning using uplink signals.

lenging requirements related to communications are beneficial for positioning as well. Some of them are dense networks, higher carrier frequencies and signal bandwidths, device-todevice communications and the use of new waveform designs.

Our focus is on how this waveform shall be designed in order to optimize the mobile terminal (MT) positioning. This paper proposes a parametric approach to define a waveform dedicated for positioning. The proposed waveform can be tuned by the MT to adapt to the current needs of accuracy and latency (time-to-first-fix) in line with the expected signalto-noise ratio (SNR). For ranging we compare the lower Cramér-Rao bound with the Ziv-Zakai bound to determine how the performances differ for the tunable parameter $\gamma$ of the waveform depending on the SNR as a key parameter to control. Fig. 1 shows our investigated scenario with three base stations (BSs) around the MT. We will provide insights how the waveform will change the performance depending on the location insight the given three BS cell structure.

The proposal of a new waveform integrates the demand of flexibility depending on the needs of the mobile terminal by parametrization the waveform to adopt it accordingly. The paper is structured as follows. In Sec. II we present the tunable waveform and two methods to bound the performance. In Sec. III we present the system model and how we evaluated the optimization of the waveform. Sec. IV presents the results.

\section{A PARAMETRIC WAVEFORM FOR POSITIONING}

The propagation delay of a radio signal can be utilized for precise ranging, which potentially meets the positioning requirement in $5 \mathrm{G}$ networks. For propagation delay-based ranging, there is a trade-off between the estimation resolution and the detection ambiguities. For a given SNR, a dedicated 


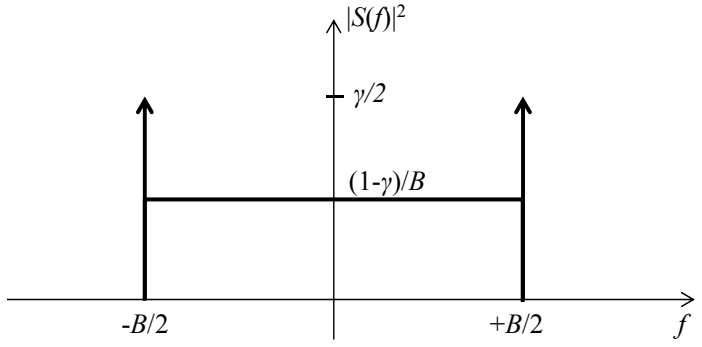

(a) Power spectrum density

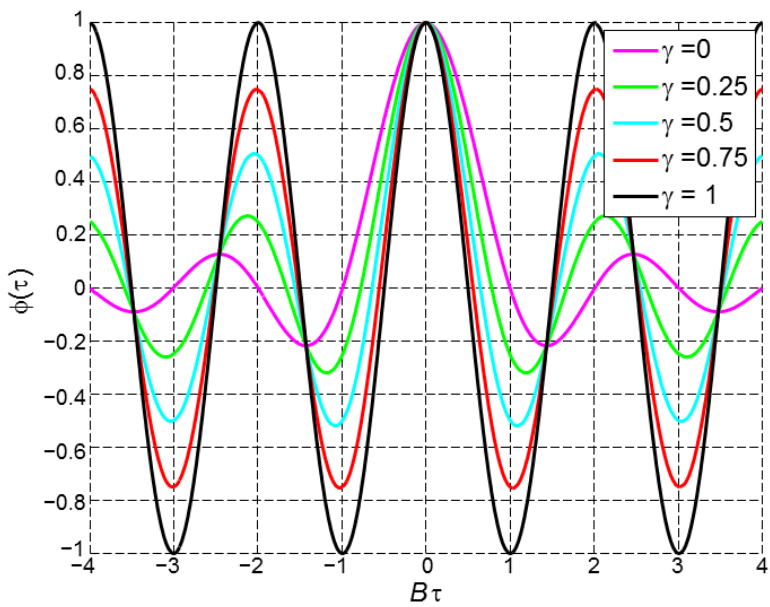

(b) Autocorrelation function

Figure 2. Dirac-rectangular waveforms

power spectrum density (PSD) exists, which minimizes the mean-square error of ranging. In this work, we investigate the impact of the PSD on positioning accuracy.

\section{A. Dirac-Rectangular Waveform}

For our investigations we consider a parameterized band limited waveform with bandwidth $B$. The waveform is built as a weighted superposition of two component signals having Dirac and rectangular PSD. The resulting PSD is

$$
|S(f)|^{2}= \begin{cases}\frac{1-\gamma}{B}+\frac{\gamma}{2}\left[\delta\left(f+\frac{B}{2}\right)+\delta\left(f-\frac{B}{2}\right)\right], & |f| \leq \frac{B}{2} \\ 0, & |f|>\frac{B}{2} .\end{cases}
$$

The corresponding autocorrelation function as the Fourier transform of the PSD calculates to

$$
\varphi(\tau)=(1-\gamma) \frac{\sin (\pi B \tau)}{(\pi B \tau)}+\gamma \cos (\pi B \tau) .
$$

Both the PSD and autocorrelation function graphs are shown in Fig. 2 for different values of the waveform parameter $\gamma$ out of the domain interval $[0,1]$. With an increasing $\gamma$, power is more concentrated at the edges of the spectrum, which leads to a tighter mainlobe and higher sidelobes for the autocorrelation function.

\section{B. Range Estimation Performance Bounds}

The Cramér-Rao lower bound (CRB) is a lower bound for the achievable variance of any unbiased estimator. For

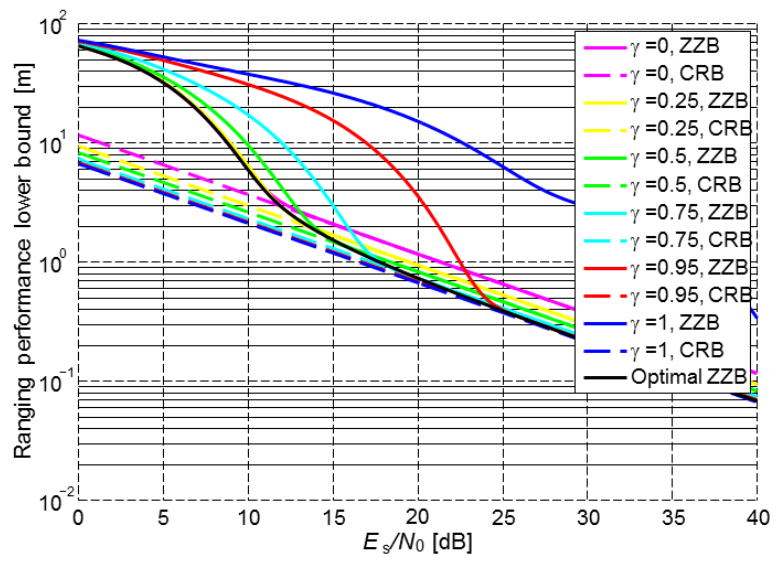

Figure 3. Square root of the ZZB and CRB for range estimation using Diracrectangular waveforms.

signal propagation delay based range estimation between a transmitter and a receiver, the $\mathrm{CRB}$ calculates to

$$
\sigma_{\mathrm{CRB}}^{2}=\frac{c_{0}^{2}}{8 \pi^{2} \beta^{2} \frac{E_{\mathrm{s}}}{N_{0}}},
$$

where $c_{0}$ is the speed of light (cf. e.g. [5]). The CRB is inverse proportional to the squared equivalent signal bandwidth $\beta^{2}$ and the signal-to-noise ratio $\frac{E \mathrm{~s}}{N_{0}}$ at the receiver. For the Dirac rectangular waveform the squared equivalent signal bandwidth is

$$
\beta^{2}=\frac{\int f^{2}|S(f)|^{2} \mathrm{~d} f}{\int|S(f)|^{2} \mathrm{~d} f}=\frac{B^{2}}{12}(1+2 \gamma), \quad 0 \leq \gamma \leq 1 .
$$

A larger $\gamma$ leads to a larger equivalent signal bandwidth, which reduces the ranging $\mathrm{CRB}$. However, it comes with the price of higher autocorrelation function sidelobes as shown in Fig. 2(b). Particularly at low SNRs, an estimator might erroneously pick the delay of the sidelobe instead of the mainlobe with non negligible probability. Due to this behavior, the estimation variance rapidly increases for lower SNRs. This threshold effect is not accounted by the CRB, which is known to be tight only for reasonably high SNRs. The Ziv-Zakai lower bound (ZZB), however, takes this effect into account. There are several forms of the ZZB in literature. For our purposes we require the ZZB for scalar parameter estimation. According to [6], the ZZB for range estimation calculates to

$$
\sigma_{\mathrm{ZZB}}^{2}=c_{0}^{2} \int_{0}^{T_{\mathrm{obs}}} \tau\left(1-\frac{\tau}{T_{\mathrm{obs}}}\right) \phi\left(\sqrt{\frac{E \mathrm{~s}}{N_{0}}(1-\varphi(\tau))}\right) \mathrm{d} \tau
$$

where

$$
\phi(x)=\frac{1}{\sqrt{2 \pi}} \int_{x}^{\infty} \mathrm{e}^{-t^{2} / 2} \mathrm{~d} t
$$

denotes the Gaussian Q-function. Parameter $T_{\text {obs }}$ describes the length of an observation interval. The signal propagation delay, as the parameter to be estimated, is equally distributed within $\left[-T_{\mathrm{obs}} / 2,+T_{\mathrm{obs}} / 2\right]$. 
The square root of the $\mathrm{CRB}$ and the $\mathrm{ZZB}$ for range estimation are shown in Fig. 3 for a signal bandwidth of $B=10 \mathrm{MHz}$ and an observation interval length of $T_{\mathrm{obs}}=$ $\frac{400 \mathrm{~m}}{c_{0}}=1.33 \mu \mathrm{s}$, which is aligned to the distance $d_{\mathrm{BS}}=400 \mathrm{~m}$ between base stations we'll use subsequently. The threshold effect mentioned above is clearly visible for the ZZBs. For increasing SNRs the ZZBs converge to the corresponding CRBs. Contrary, the SNR value at which the ZZB starts to diverge from the $\mathrm{CRB}$ increases with increasing squared equivalent bandwidth $\beta^{2}$. For the Dirac waveform, i.e. $\gamma=1$, the ZZB shows no convergence to the corresponding CRB. The autocorrelaton function equals to a cosine and the amplitude of the sidelobes are equal to the mainlobe amplitude. Therefore, the autocorrelation function is ambiguous in its largest amplitude such that an algorithm has equal probability in estimating the delay of a sidelobe instead of a mainlobe.

Minimizing the ZZB with respect to the waveform parameter $\gamma$ leads to an optimal ZZB which is drawn in Fig. 3. This optimum is the lower envelope curve of the ZZB graphs for all $\gamma \in[0,1]$.

The results above indicate that there is a tradeoff between the ranging performances at high and medium to low SNRs. Depending on the available signal power respectively the receiver SNR we might decide for an optimum spectrum form. However, this depends on the local distribution of mobile terminals. Therefore, it is beneficial to keep a $5 \mathrm{G}$ positioning waveform flexible with respect to its power spectrum density.

\section{Positioning Performance Evaluation}

\section{A. Signal Model}

We investigate an uplink scenario, where a MT transmits a signal $s(t)$ as shown in Fig. 1. At the base stations (BSs) we receive the signals

$$
r_{i}(t)=s_{i}\left(t-\tau_{i}\right)+n_{i}(t), \quad i=1, \ldots, N_{\mathrm{BS}},
$$

which consist of the delayed and attenuated transmit signal and additive white Gaussian noise (AWGN) $n_{i}(t)$ with noise power density $N_{0}$. The observed propagation delays

$$
\tau_{i}(\boldsymbol{\theta})=\frac{d_{i}}{c_{0}}+T_{\mathrm{MT}}=\frac{1}{c_{0}} \sqrt{\left(x-x_{i}\right)^{2}+\left(y-y_{i}\right)^{2}}+T_{\mathrm{MT}}
$$

depend on the distance $d_{i}$ between the MT and $\mathrm{BS}_{i}$. With $\tau_{i}$ we also consider an unknown time offset $T_{\mathrm{MT}}$ between the MT and the BSs. The BSs itself are assumed to be synchronized. The unknown variables to be estimated, i.e., the position and time offset of the MT, are collected in a parameter vector $\boldsymbol{\theta}=\left[x, y, T_{\mathrm{MT}}\right]^{\mathrm{T}}$. We assume line-of-sight free space signal propagation conditions. Therefore, the signal-to-noise ratios (SNRs)

$$
\mathrm{SNR}_{i}=\frac{E \mathrm{~s}_{i}}{N_{0}}=\frac{P_{\mathrm{S}_{i}}}{P_{\text {noise }}}=\frac{P_{\mathrm{TX}} G_{\mathrm{TX}} G_{\mathrm{RX}}\left(\frac{c_{0}}{4 \pi f_{\mathrm{C}} d_{i}}\right)^{2}}{k_{\mathrm{B}} \vartheta B}
$$

observed at the BSs depend on the TX power $P_{\mathrm{TX}}$, the antenna gains $G_{\mathrm{TX}}$ and $G_{\mathrm{RX}}$ at the MT and BSs, carrier frequency $f_{\mathrm{C}}$, signal bandwidth $B$ and the distance $d_{i}$ between the MT and $\mathrm{BS}_{i}$. The noise power density $N_{0}=k_{\mathrm{B}} \vartheta$ is calculated from the Boltzmann constant $k_{\mathrm{B}}$ and the system noise temperature $\vartheta$ (cf. Table I).

\section{B. Fisher Information for Positioning}

For the evaluation of the positioning performance of the MT we start with the calculation of the CRB for vector parameter estimation [5]. The unknown parameters which we wish to estimate are the position and time base offset of the MT, which we collect in a vector $\boldsymbol{\theta}=\left[x, y, T_{\mathrm{MT}}\right]^{\mathrm{T}}$. For the calculation of the $\mathrm{CRB}$ we require the Fisher information matrix. Its components

$$
\begin{array}{r}
\mathbf{F}_{k, \ell}=\mathrm{E}\left\{\left(\frac{\partial}{\partial \theta_{k}} \log \mathrm{p}(\mathbf{r}(t) \mid \boldsymbol{\theta})\right)\left(\frac{\partial}{\partial \theta_{\ell}} \log \mathrm{p}(\mathbf{r}(t) \mid \boldsymbol{\theta})\right)\right\} \\
=\frac{2}{N_{0}} \operatorname{Re} \int_{-\infty}^{+\infty} \sum_{i=1}^{N_{\mathrm{BS}}}\left(\frac{\partial}{\partial \theta_{k}} s_{i}\left(t-\tau_{i}(\boldsymbol{\theta})\right)\right) \\
\left(\frac{\partial}{\partial \theta_{\ell}} s_{i}^{*}\left(t-\tau_{i}(\boldsymbol{\theta})\right)\right) \mathrm{d} t
\end{array}
$$

are calculated from the likelihood function which in case of AWGN can be expressed as

$\mathrm{p}(\mathbf{r}(t) \mid \boldsymbol{\theta}) \propto \exp \left(-\frac{1}{N_{0}} \sum_{i=1}^{N_{\mathrm{BS}}} \int_{-\infty}^{+\infty}\left|s_{i}\left(t-\tau_{i}(\boldsymbol{\theta})\right)-r_{i}(t)\right|^{2} \mathrm{~d} t\right)$.

For notational convenience we omit constant factors. These factors will vanish when calculating derivatives of the logarithmic likelihood function according to Eq. (10). The likelihood function provides the conditional probability density of observing the signals $\mathbf{r}(t)=\left[r_{1}(t), \ldots, r_{N_{\mathrm{BS}}}(t)\right]^{\mathrm{T}}$ at the BSs given the MT position and time offset collected in $\boldsymbol{\theta}=\left[x, y, T_{\mathrm{MT}}\right]^{\mathrm{T}}$. In matrix notation the Fisher information matrix

$$
\mathbf{F}=c_{0}^{2} \mathbf{J}_{\boldsymbol{\tau}}^{\mathrm{T}} \operatorname{diag}\left(\sigma_{\mathrm{CRB} 1}^{-2}, \ldots, \sigma_{\mathrm{CRB} N_{\mathrm{BS}}}^{-2}\right) \mathbf{J}_{\boldsymbol{\tau}}
$$

consists of the Jacobian matrix $\mathbf{J}_{\boldsymbol{\tau}}=\frac{\partial \boldsymbol{\tau}}{\partial \boldsymbol{\theta}}$ for the delay vector $\boldsymbol{\tau}=\left[\tau_{1}(\boldsymbol{\theta}), \tau_{2}(\boldsymbol{\theta}), \tau_{3}(\boldsymbol{\theta})\right]^{\mathrm{T}}$ and a diagonal matrix containing the inverse ranging $\mathrm{CRBs}$ according to Eq. (3). Matrix $\mathbf{G}=c_{0} \mathbf{J}_{\boldsymbol{\tau}}$ is also called the geometry matrix. It only depends on the MT and BSs positions relative to each other. This matrix does depend neither on the signal we have transmitted at the MT nor on the SNR. The dependency on the signal and propagation properties is solely contained in the diagonal matrix $\operatorname{diag}\left(\sigma_{\mathrm{CRB} 1}^{-2}, \ldots, \sigma_{\mathrm{CRB} N_{\mathrm{BS}}}^{-2}\right)$. The CRB is the inverse of the Fisher information matrix and contains lower bounds for the variance of any unbiased estimation of the unknown parameters - in our case $\boldsymbol{\theta}=\left[x, y, T_{\mathrm{MT}}\right]^{\mathrm{T}}-$ in its main diagonal.

\section{Waveform Optimization}

The CRB for ranging, as introduced in Sec. II-B and contained in Eq. (12), is known to be loose for low SNRs. This bound is monotonically decreasing with increasing squared equivalent bandwidth $\beta^{2}$, or equivalently, increasing parameter $\gamma$ for all SNRs. Minimizing the CRB with respect to the waveform parameter $\gamma$ results in an optimal waveform parameter $\gamma_{\text {opt }}=1$, independent of the SNRs, and therefore, the MT position. The ZZB, however, does account for the threshold effect as shown in Fig. 3. 
Table I

SYSTEM PARAMETERS.

\begin{tabular}{lll}
\hline Parameter & & Value \\
\hline Carrier frequency & $f_{\mathrm{C}}$ & $5 \mathrm{GHz}$ \\
Effective power & $P_{\mathrm{TX}} \cdot G_{\mathrm{TX}} \cdot G_{\mathrm{RX}}$ & $10 \mathrm{dBm}$ \\
Signal bandwidth & $B$ & $10 \mathrm{MHz}$ \\
Noise power density & $N_{\mathrm{O}}=k_{\mathrm{B}} \vartheta$ & $N_{0}=-173.8 \mathrm{dBm} / \mathrm{Hz}$ \\
Boltzmann constant & $k_{\mathrm{B}}$ & $1.381 \cdot 10^{-23} \mathrm{Ws} / \mathrm{K}$ \\
Noise temperature & $\vartheta$ & $300 \mathrm{~K}$ \\
Base station distance & $d_{\mathrm{BS}}$ & $400 \mathrm{~m}$ \\
\hline
\end{tabular}

As an approach for optimizing the positioning performance with respect to the waveform parameter $\gamma$ we replace the CRB ranging variances in Eq. (12) with the corresponding ZZB obtained from Eq. (5) and get

$$
\tilde{\mathbf{F}}=c_{0}^{2} \mathbf{J}_{\boldsymbol{\tau}}^{\mathrm{T}} \operatorname{diag}\left(\sigma_{\mathrm{ZZB} 1}^{-2}, \ldots, \sigma_{\mathrm{ZZB} N_{\mathrm{BS}}}^{-2}\right) \mathbf{J}_{\boldsymbol{\tau}}
$$

as a kind of modified Fisher information matrix. Its inverse

$$
\tilde{\mathbf{C}}=\tilde{\mathbf{F}}^{-1}
$$

provides $2^{\text {nd }}$ order moments for the estimation error of the unknown parameters on its main diagonal. However, these values are formally no lower bounds but provide an easy to calculate cost function for waveform optimization. Similar to the CRB approach we use the square root

$$
\sigma_{\operatorname{pos}}(\gamma)=\sqrt{\tilde{\mathbf{C}}_{1,1}+\tilde{\mathbf{C}}_{2,2}}
$$

of first two main diagonal elements of matrix $\tilde{\mathbf{C}}$ as a measure for the expectable position estimation variance. This metric depends on the ZZB ranging variances $\sigma_{\mathrm{ZZB} i}^{2}$. Since the optimal choice of the waveform parameter $\gamma$ is dependent on the SNR, we can expect that there is also an optimum

$$
\gamma_{\mathrm{opt}}=\underset{0 \leq \gamma \leq 1}{\arg \min } \sigma_{\mathrm{pos}}(\gamma)
$$

leading to an optimal positioning variance

$$
\sigma_{\mathrm{opt}}=\sigma_{\mathrm{pos}}\left(\gamma_{\mathrm{opt}}\right) \text {. }
$$

The optimal positioning variance depends on the position of the MT as well as on further system parameters like the base station distance, effective power, etc.

\section{RESults}

We consider an uplink transmission scenario as shown in Fig. 1 and evaluate the positioning performance for a MT in the area between 3 BSs. The system parameters for positioning performance evaluation are summarized in Table I. As a reference we consider a signal, transmitted at the MT and received at the BSs, with uniform (rectangular) PSD. This means we choose $\gamma=0$ for the Dirac-rectangular waveform introduced in Sec. II-A. Reference signals, which are currently used in today's mobile radio systems, typically show uniform power distribution over the spectrum. For a chosen position of the MT we calculate the SNRs observed at the BSs according to Eq. (9). From these SNRs we obtain the $\mathrm{ZZB}$ for ranging according to Eq. (5). The ZZB values are plugged into Eq. (13). Using Eqs. (14) and (15) we finally calculate the positioning error $\sigma_{\text {pos }}(0)$ for our reference waveform $(\gamma=0)$. Fig. 4 shows the positioning error for each position of the MT in the considered environment as a color plot. We achieve the best performance in the center of the area between the BSs. Here we obtain SNR values with similar order of magnitude. Leaving this center area the obtained SNRs get more and more unbalanced. This leads to worse performance since the positioning error is dominated by the lower SNR values obtained at BSs at higher distances.

As a next step we minimize the positioning error according to Eq. (16) for each MT position. The optimal choice for the waveform parameter $\gamma$ is shown in Fig. 5. Again, due to similar SNR levels at the center area we can choose waveforms which provide better performance at higher SNRs, i.e., higher $\gamma$. For our environment we obtain $\gamma_{\text {opt }} \approx 0.8$ at the center area. This means that the optimum waveform spends approximately $80 \%$ of the available power at the spectrum edges. The remaining $20 \%$ are distributed uniformly over the spectrum range in between. Leaving the center area we more and more obtain unbalanced SNR values. Since the positioning error is significantly determined by the lower SNRs, we have to adapt the waveform such that we obtain a reasonable ranging performance at these lower SNRs. When more and more veering the area between the BSs, all SNR values decrease and the optimum waveform parameter approaches zero, i.e., $\gamma_{\text {opt }} \rightarrow 0$ which means a rectangular PSD. The optimal positioning performance obtained from the optimal waveform parameter $\gamma_{\mathrm{opt}}$ according to Eq. (17) is shown in Fig. 6. Compared to the reference waveform, the positioning error decreases mainly at the center area. For comparison we calculate the performance gain as

$$
\text { Gain }=\frac{\sigma_{\mathrm{pos}}(0)-\sigma_{\mathrm{opt}}}{\sigma_{\mathrm{pos}}(0)}=1-\frac{\sigma_{\mathrm{opt}}}{\sigma_{\mathrm{pos}}(0)}
$$

for the optimal waveform choice compared to the reference waveform with rectangular PSD. As shown in Fig. 7 we observe the highest gain of $37.3 \%$ at the center area. As already discussed the gain approaches zero when leaving the area between the BSs.

When decreasing the BS distance $d_{\mathrm{BS}}$ or increasing the effective power $P_{\mathrm{TX}} \cdot G_{\mathrm{TX}} \cdot G_{\mathrm{RX}}$ we obtain higher SNR levels at the center area, leading to a higher gain. The maximum achievable gain for the Dirac-rectangular waveform is achieved if the SNR values approach infinity. Therefore, we may expect that $\gamma_{\text {opt }} \rightarrow 1$. The maximum achievable gain can be calculated as

$$
\begin{aligned}
\operatorname{Gmax} & =1-\frac{\sigma_{\mathrm{pos}}(1)}{\sigma_{\mathrm{pos}}(0)}=1-\sqrt{\frac{\sigma_{\mathrm{CRB}}^{2}(\gamma=1)}{\sigma_{\mathrm{CRB}}^{2}(\gamma=0)}} \\
& =1-\sqrt{\frac{\beta_{\mathrm{CRB}}^{2}(\gamma=0)}{\beta_{\mathrm{CRB}}^{2}(\gamma=1)}}=1-\frac{1}{\sqrt{3}}=42,3 \%
\end{aligned}
$$

using Eqs. (3) and (4).

\section{Summary AND OUTLOOK}

Today's mobile radio systems deploy reference signals which are used for signal propagation delay-based mobile terminal positioning. Usually the signal power of such reference 


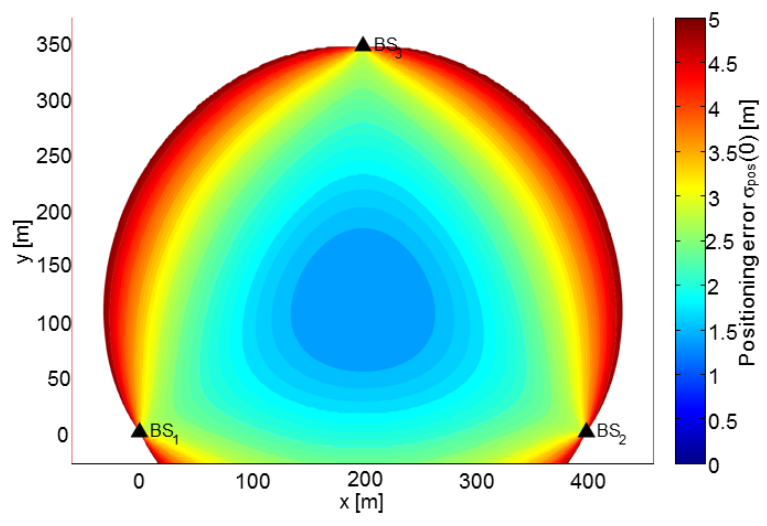

Figure 4. Positioning error $\sigma_{\mathrm{pos}}(0)$ for a waveform with rectangular PSD $(\gamma=0)$.

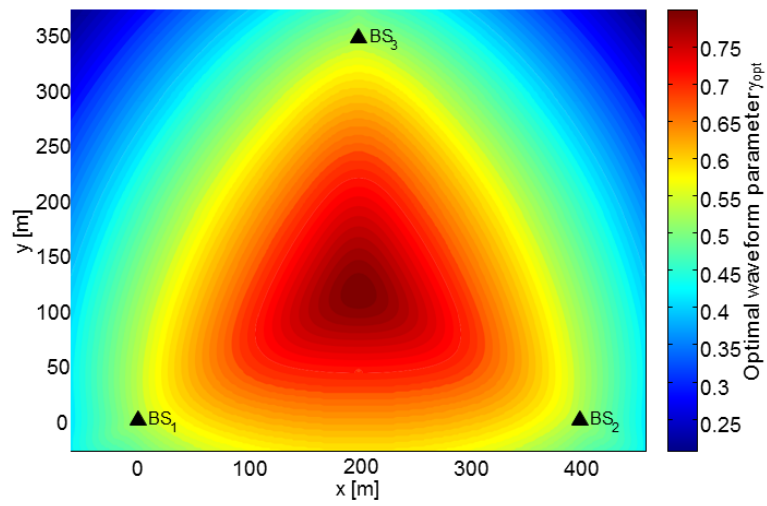

Figure 5. Optimal waveform parameter $\gamma_{\text {opt }}$ for the Dirac-rectangular waveform

signals is uniformly distributed over the available spectrum. Generally, a uniform power distribution is not optimal. We have introduced the Dirac-rectangular waveform as a parametric waveform in order to adjust and optimize the power spectrum of reference signals which are used for propagation delay estimation. For optimization we have derived a cost function which combines the approaches of the Cramér-Rao and Ziv-Zakai lower bounds for position and propagation delay estimation. In this paper we have considered mobile terminal positioning in an area surrounded by 3 base stations. The Dirac-rectangular waveform has been used in the uplink case in order to estimate the signal propagation delays at each base station. Investigations for an exemplary environment with a pairwise base station distance of $d_{\mathrm{BS}}=400 \mathrm{~m}$ have shown that an optimized waveform choice can decrease the positioning error by $37.3 \%$ at the center of the area between the base stations compared to a state-of-the art reference signal with uniform power spectrum density. When increasing the observed signal-to-noise ratios at the base stations, e.g. by increasing the transmit power at the mobile terminal or decreasing the base station distance, this gain can become up to $42.3 \%$.

For our investigations, we have considered a waveform which can be controlled by one scalar parameter. Further evaluations will generalize the domain of waveforms which are

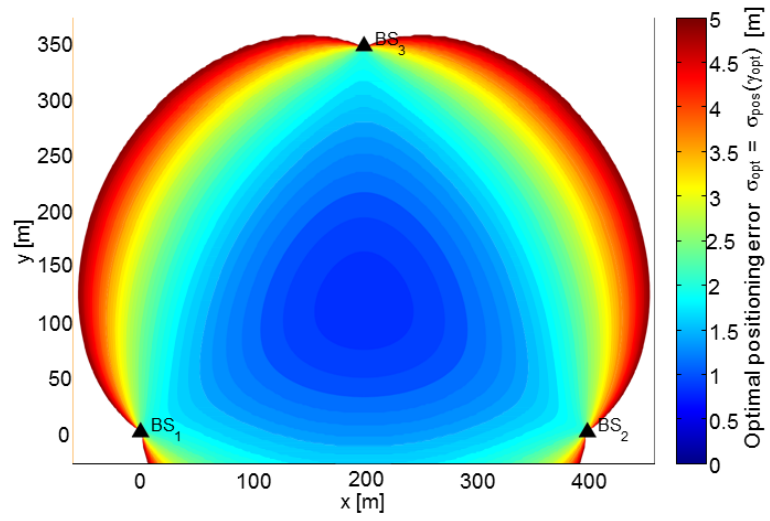

Figure 6. Optimal positioning error $\sigma_{\mathrm{opt}}=\sigma_{\mathrm{pos}}\left(\gamma_{\mathrm{opt}}\right)$ for the Diracrectangular waveform.

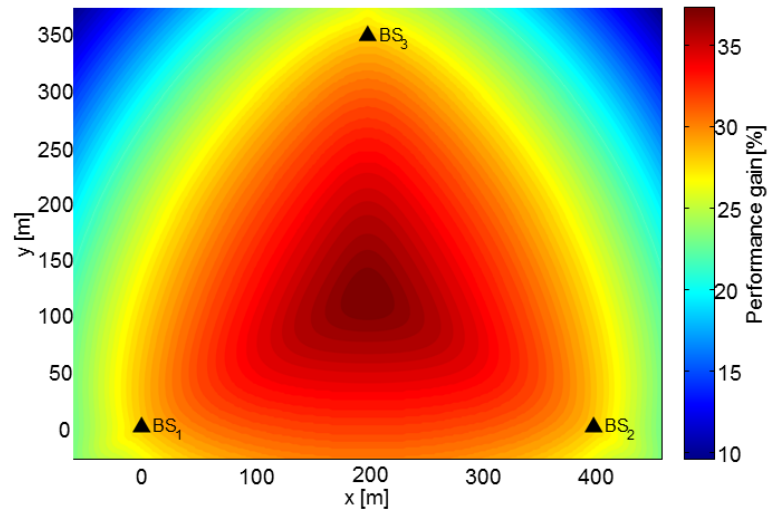

Figure 7. Performance gain $1-\sigma_{\mathrm{opt}} / \sigma_{\mathrm{pos}}(0)$ for the optimal waveform choice compared to a reference waveform with rectangular PSD.

available for optimization, include more complex signal propagation models and consider cooperative positioning methods.

\section{ACKNOWLEDGMENT}

This work was partially supported by the EU project HIGHTS (High precision positioning for cooperative ITS applications) MG-3.5a-2014-636537 and the DLR project Dependable Navigation.

\section{REFERENCES}

[1] G. Fettweis and S. Alamouti, "5G: Personal mobile internet beyond what cellular did to telephony," IEEE Communications Magazine, vol. 52, no. 2, pp. 140-145, Feb. 2014.

[2] J. Andrews, S. Buzzi, W. Choi, S. Hanly, A. Lozano, A. Soong, and J. Zhang, "What will 5G be?" IEEE Journal on Selected Areas in Communications, vol. 32, no. 6, pp. 1065-1082, Jun. 2014.

[3] A. Osseiran, F. Boccardi, V. Braun, K. Kusume, P. Marsch, M. Maternia, O. Queseth, M. Schellmann, H. Schotten, H. Taoka, H. Tullberg, M. Uusitalo, B. Timus, and M. Fallgren, "Scenarios for 5G mobile and wireless communications: The vision of the METIS project," IEEE Communications Magazine, vol. 52, no. 5, pp. 26-35, May 2014.

[4] "5G White Paper," Dec. 2014, version 1.0, Final Executive Version. [Online]. Available: http://www.ngmn.org/uploads/media/ 141222_NGMN-Executive_Version_of the_5G_White_Paper_v1_0.pdf

[5] S. M. Kay, Fundamentals of Statistical Signal Processing - Estimation Theory. Prentice Hall, 1993.

[6] C. Musso and J.-P. Ovarlez, "Improvement of the Ziv-Zakai lower bound for time delay estimation," in 15th European Signal Processing Conference (EUSIPCO 2007), Poznan, Poland, Sep. 2007, pp. 960-964. 